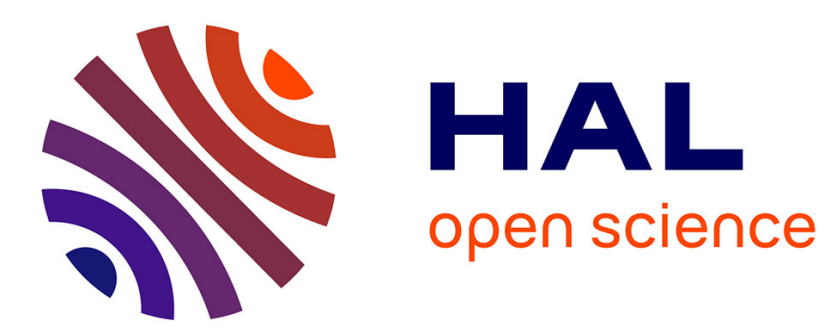

\title{
Ultimate efficiency of spectral beam combining by volume Bragg gratings
}

L.B. Glebov, Derrek R. Drachenberg, Oleksiy Andrusyak, George Venus, Vadim Smirnov, Julien Lumeau, Leonid B. Glebov

\section{To cite this version:}

L.B. Glebov, Derrek R. Drachenberg, Oleksiy Andrusyak, George Venus, Vadim Smirnov, et al.. Ultimate efficiency of spectral beam combining by volume Bragg gratings. Applied optics, 2013, 52 (30), pp.7233-7242. hal-00945858

\section{HAL Id: hal-00945858 https://hal.science/hal-00945858}

Submitted on 26 Mar 2019

HAL is a multi-disciplinary open access archive for the deposit and dissemination of scientific research documents, whether they are published or not. The documents may come from teaching and research institutions in France or abroad, or from public or private research centers.
L'archive ouverte pluridisciplinaire HAL, est destinée au dépôt et à la diffusion de documents scientifiques de niveau recherche, publiés ou non, émanant des établissements d'enseignement et de recherche français ou étrangers, des laboratoires publics ou privés. 


\title{
Ultimate Efficiency of Spectral Beam Combining by Volume Bragg Gratings
}

\author{
Derrek R. Drachenberg, ${ }^{1,2 *}$ Oleksiy Andrusyak, ${ }^{1,3}$ George Venus, ${ }^{1}$ Vadim Smirnov, ${ }^{4}$ Julien Lumeau, ${ }^{1,5}$ \\ Leonid B. Glebov, 1,4 \\ ${ }^{1}$ CREOL, College of Optics and Photonics, University of Central Florida, 4000 Central Florida Blvd., Orlando, FL 32816 \\ ${ }^{2}$ Currently with NIF-Photon Science and Applications, Lawrence Livermore National Lab 7000 East Ave., Livermore, CA \\ 94551 \\ ${ }^{3}$ Currently with IPG Photonics, 50 Old Webster Rd., Oxford, MA 01540 \\ ${ }^{4}$ OptiGrate Corp, 562 South Econ Circle, Oviedo, FL 32765-4311 \\ ${ }^{5}$ Currently with Institut Fresnel, 52-Avenue Escadrille Normandie Niemen 13013, Marseille, France \\ *Corresponding author: drachenberg1@llnl.gov
}

Received Month X, XXXX; revised Month X, XXXX; accepted Month X,

XXXX; posted Month X, XXXX (Doc. ID XXXXX); published Month X, XXXX

\begin{abstract}
Spectral beam combining (SBC) by volume Bragg gratings (VBGs) recorded in photo-thermo-refractive (PTR) glass is a powerful tool for laser applications which require higher radiance than a single laser unit can achieve. The beam combining factor (BCF) is introduced as a tool to compare various beam combining methods and experiments. It describes the change of radiance provided by a beam combining system but is not affected by the initial beam quality of the combined lasers. A method of optimization of VBGs providing the maximum efficiency of spectral beam combining has been described for an arbitrary number of beams. An experiment confirming the proposed modeling for a two beam SBC system by a single VBG has demonstrated a total combined power of $301 \mathrm{~W}$ with a channel separation of $0.25 \mathrm{~nm}$, combining efficiency of $97 \%$, close to diffraction limited divergence with $\mathrm{M}^{2}=1.18$, beam combining factor $(\mathrm{BCF})$ of 0.77 , and spectral radiance of $770 \mathrm{TW} /\left(\mathrm{sr} \cdot \mathrm{m}^{2} \cdot \mathrm{nm}\right)$, the highest to date for SBC.
\end{abstract}

OCIS codes: $230.1480,090.2890,140.3298,140.3510,160.2750$

\section{INTRODUCTION}

Fundamental nonlinear and thermal limits on the average power that can be achieved by a single laser source are leading researchers into beam combining to achieve greater continuouswave laser power. For applications in which high intensity on the target are desired, the goal of beam combining cannot simply be to increase the power, but to increase the radiance (brightness) which is defined as the beam power divided by the product of the beam waist area and the solid angle in the far field. High power beam combining that increases brightness in comparison to that of original beams can be broadly categorized into two approaches, coherent and incoherent beam combining [1]. Incoherent beam combining can be accomplished by the use of an angularly dispersive element such as a surface diffraction grating [2,3], or a prism [4], or by a spectral filtering (dichroic) element such as thin film filters [5] or volume Bragg gratings (VBGs) [6-9]. Other incoherent methods of beam combining such as incoherent arrays, fiber bundles, or beam transformation lenses, are not capable of increasing the radiance of the combined beam over that of a single beam according to the radiance theorem which states that the combined spectral radiance of a set of mutually incoherent but otherwise identical beams cannot be increased over that of the highest radiance beam [10]. It should be emphasized that while spectral beam combining increases spatial brightness, the spectral brightness of a combined beam is decreased approximately by the ratio of the spectral width of an original beam to the spectral distance between combined beams. This paper focuses on the important design optimization and performance metrics of VBGs used for spectral beam combining systems.

VBGs in photo-thermo-refractive (PTR) glass have been used as combining elements in high power spectral beam combining (SBC) for more than a decade [11]. SBC is achieved by diffracting one beam from a VBG at the Bragg wavelength with near $100 \%$ efficiency while transmitting another beam at a nearby wavelength not equal to the Bragg wavelength. Coupled wave theory for thick holograms, also called volume Bragg gratings, was thoroughly developed by Kogelnik's 1969 [12] paper. Subsequent papers on the topic have attempted to exploit a novel type of VBG, method of manufacture, or to describe them in the context of simplifying the theory to create a set of equations that may be used by engineers [13-17]. To our knowledge, no paper has been published describing VBG parameters required for maximum brightness in a combined beam.

In this paper it is proposed that an increase in brightness is the important metric when describing the quality of a beam combining system, and we therefore will introduce the Beam Combining Factor. When modeling VBGs for SBC, a balance must be found between increasing the reflection at the Bragg wavelength $(\mathrm{s})$ and increasing the transmission at the transmission wavelength(s). A method of maximizing efficiency 
in VBG SBC systems with an arbitrary number of beams is described. Some examples are demonstrated that illustrate the existence of VBG parameters that enable an absolute maximum in beam combining efficiency. Finally ultra-high spectral radiance (SBC) of two fiber lasers by VBGs, demonstrating 0.25 $\mathrm{nm}$ spectral separation between beams, combined output power of $301 \mathrm{~W}, 97 \%$ combining efficiency, and a spectral radiance of $783 \mathrm{TW} /\left(\mathrm{sr}^{2} \mathrm{~m}^{2} \cdot \mathrm{nm}\right)$, is reported, the highest to date for an SBC system.

\section{THE BEAM COMBINING FACTOR (BCF)}

Radiance is an important and appropriate metric for characterizing the ability of a beam to deliver high intensity on a target, either at the waist of a focused beam or at the center of a collimated beam after propagation through the atmosphere.

Radiance, also called spatial brightness, is defined as the power (P) divided by the product of the solid angle in the far field and the area of the beam waist. The spatial brightness for a radially symmetric beam is given by [1],

$$
\begin{aligned}
B & =\frac{P}{\pi \omega^{2} \pi \theta_{\text {Beam }}{ }^{2}}=\frac{P}{\pi \omega^{2} \pi \theta_{\text {ideal_Gaussian }}^{2}\left(M^{2}\right)^{2}} \\
& =\frac{P}{\pi \omega^{2} \pi\left(\frac{\lambda}{\pi \omega}\right)^{2}\left(M^{2}\right)^{2}}=\frac{P}{\lambda^{2}\left(M^{2}\right)^{2}}
\end{aligned}
$$

where $\Pi \omega^{2}$ is the area of the beam waist, $\Pi \theta^{2}$ is the solid angle in far field, $\Pi \theta_{\text {ideal }}{ }^{2}$ is the solid angle in far field for a Gaussian beam, and the $\mathrm{M}^{2}$ beam quality factor is defined as the measured beam far field divergence divided by the far field divergence of an ideal Gaussian beam with an equivalent beam waist size.

$$
M^{2}=\frac{\theta}{\lambda /(\pi \omega)}=\frac{\theta_{\text {beam }}}{\theta_{\text {idea-Gaussian }}}
$$

It should be emphasized that while the brightness is proportional to the beam power, it is inversely proportional to the square of the $\mathrm{M}^{2}$ beam quality factor. Therefore, for the purposes of obtaining high brightness combined beams, it is important to maintain not only high efficiency of combining that provides maximum power in an output beam but to keep high beam quality through the combining system. This means that combining system optimization should be based on a tradeoff between combining efficiency and beam quality.

Another important consideration results from the finite widths of atmospheric transparency windows where a beam is intended to propagate. For example, the spectral width of a high transparency window near $1 \mu \mathrm{m}$ in low atmosphere is only 50 $\mathrm{nm}$. This means that a spectral width of a combined beam should not exceed it. Therefore, when characterizing a laser system operating in an absorbing medium it is necessary to consider the spectral brightness which is defined as the spatial brightness per unit wavelength, $(B / \Delta \lambda)$. Spectral brightness then becomes the figure of merit for a system designed for this purpose. With equations (1) and (2), the beam combining system can be characterized in a meaningful way.

The goal of both coherent and spectral beam combining systems is to increase the brightness, and the quality of beam combining of various systems can be compared by calculating the ratio of the output radiance with the sum of the input radiances. If only the beam combining portion of the laser system is to be characterized, the radiance alone cannot accomplish this due to the fact that this also includes characterization of the input beams. Therefore, a metric is needed which considers only the quality of beam combining. Proposed here is the beam combining factor (BCF) which is defined as the brightness of the combined beam divided by the sum of the brightness values of the input beams.

$$
B C F=\frac{B_{\text {combined }}}{\sum B_{\text {unit }}}=\frac{\left[P /\left(M^{2}\right)^{2}\right]_{\text {combined }}}{\sum\left[P /\left(M^{2}\right)^{2}\right]_{\text {unit }}}
$$

It is important that the $\mathrm{BCF}$ characterizes a beam combining system but not the original sources of radiation and, therefore, its value does not depend on the quality of the input beams. The $\mathrm{BCF}$ ranges from $0-1$. If the power of each beam is added into the final beam without any loss and without any degradation of the beam quality factor, $\mathrm{M}^{2}$, then the $\mathrm{BCF}$ will be 1 . A further simplification can be made to the $\mathrm{BCF}$ equation if the input beams are assumed to be equal in power and beam quality. In this case, the BCF depends only on the system efficiency and the ratio between the input beam $\mathrm{M}^{2}$ factor and the resulting combined beam $\mathrm{M}^{2}$ factor.

$$
B C F=\eta_{B C} \frac{\left(M^{2}\right)_{\text {unit }}^{2}}{\left(M^{2}\right)_{\text {combined }}^{2}}
$$

One can see that BCF is determined by both losses of power and beam quality degridation in a combining system. In this paper we will discuss the problem of efficiency in connection with spectral properties of VBGs while beam quality deteriorations caused by grating imperfections and its interaction with high power laser beams will be described in following papers.

\section{MODELING OF SBC BY VBGs}

\section{A.VBG Diffraction Efficiency for Monochromatic Plane Waves}

A method for optimizing a spectral beam combining system with an arbitrary number of channels is to be developed for real laser beams. Kogelnik's coupled wave theory for thick holograms does not take into account divergence and spectral bandwidth of diffracted beams. However, it is clear that the diffraction efficiency of a beam passing through a VBG will change depending on beam divergence and spectral bandwidth. A full description of modeling the diffraction efficiency spectrum for a single reflecting grating including the effects of laser beam divergence and spectral bandwidth has been published [17]. The important resulting equations that are needed to perform optimization are given here. The diffraction efficiency as a 
function of beam angle or wavelength for use with a single frequency infinite plane wave is given in equation (5).

$$
\eta(\xi)=\frac{\sin ^{2}\left(\sqrt{\xi^{2}-S^{2}}\right)}{\xi^{2} / S^{2}-\cos ^{2}\left(\sqrt{\xi^{2}-S^{2}}\right)}
$$

where the grating strength $\mathrm{S}$ for an unslanted grating is,

$$
S_{0}=\frac{2 \pi n_{a v} t \delta n}{\lambda_{0} f}
$$

the Bragg condition dictates,

$$
f=2 n_{a v} \frac{\cos \left(\theta_{m}\right)}{\lambda_{0}}
$$

And finally the detuning parameter is given by,

$$
\begin{gathered}
\xi(d \theta, \Delta \lambda)=\left(\frac{2 \pi n_{a v} t \cos \left(\theta_{m}\right)}{\lambda_{0}}\right) \\
\times\left(d \theta \frac{\sin \left(\theta_{m}\right)}{\cos \left(\theta_{m}\right)}+\frac{d \theta^{2}}{2}+\frac{\Delta \lambda}{\lambda_{0}}\right)
\end{gathered}
$$

In the above equations described explicitly in Ref. [17], $\mathrm{n}_{\mathrm{av}}$ is the average refractive index of a photosensitive material used for hologram recording, $\mathrm{t}$ is the thickness of a VBG, $\mathrm{n} n$ is the refractive index modulation, $\lambda_{0}$ is the Bragg wavelength, $\theta_{\mathrm{m}}$ is the angle between the grating vector and incident beam wave vector in medium, and $d \theta$, and $\Delta \lambda$ are the detuning variables in angle and wavelength respectively. In this paper, we will not provide an illustration of calculation in generalized form but demonstrate the results with examples that could be achieved in experiments. An example of the diffraction efficiency as a function of wavelength for a plane-wave monochromatic beam is shown in Figure 1 (blue dots) for a VBG with a thickness of $4.7 \mathrm{~mm}$, and refractive index modulation of $320 \mathrm{ppm}$. The grating period is $358.9 \mathrm{~nm}$. The Bragg condition is satisfied at $1064 \mathrm{~nm}$ for an incident angle of $4^{\circ}$ angle in air. Figure 2 shows the experimental architecture for spectral beam combining using a reflecting VBG. More than two beams are combined by cascading multiple VBGs, each diffracting one beam and transmitting all previously combined beams.

\section{B. Optimization of VBG Parameters for Single Stage SBC of Monochromatic Plane Waves}

Beam combining efficiency, $\eta_{\mathrm{BC}}$, is determined by both diffraction efficiency of the diffracted beams and losses of the beams transmitted through the VBGs. Diffraction efficiency for a particular Bragg wavelength and Bragg angle can be increased by increasing $S$ which is proportional to the product of thickness and refractive index modulation, $t \times \delta n$. Increasing thickness has the effect of increasing the peak in diffraction efficiency while narrowing the bandwidth angularly and spectrally. Increasing the refractive index modulation has the effect of increasing the peak in diffraction efficiency at the central Bragg wavelength and in the side lobes combined with widening of the spectral and angular selectivity. These two effects can be seen from Ref. [17] Figures 3 and $4 \mathrm{~b}$.

This is why the desired optimization is on beam combining efficiency which means high diffraction at the Bragg wavelength while obtaining high transmission at a nearby transmission wavelength. To optimize a two beam SBC system, henceforth single stage SBC system, produced by a single VBG, a spectral separation between beams, and angular divergence for the beam should be chosen. Let us assume for the first approximation that the VBG interacts with monochromatic plane waves, and that the VBG does not have material losses (absorption and scattering). These assumptions will be reconsidered later. Given such parameters, a set of diffraction efficiency spectra, $\eta(\Delta \lambda, t, \delta n)$, can be calculated for a range of thickness and refractive index modulations, where $\Delta \lambda$ is the detuning parameter in wavelength, $t$, is the VBG thickness, and $\delta n$ is the refractive index modulation. For each calculated diffraction efficiency spectrum, the beam combining efficiency should be determined by the following equation,

$$
\begin{aligned}
& \eta_{B C}(t, \delta n)=\frac{1}{P_{B}+P_{T 1}} \\
& \times\left\{P_{B}\left[\eta\left(\lambda_{B}, t, \delta n\right)\right]+P_{T 1}\left[1-\eta\left(\lambda_{T 1}, t, \delta n\right)\right]\right\}
\end{aligned}
$$

where $\lambda_{\mathrm{B}}$ is the Bragg wavelength, and $\lambda_{\mathrm{T} 1}$ is the transmission wavelength such that $\lambda_{\mathrm{B}}-\lambda_{\mathrm{T} 1}=\Delta \lambda_{\mathrm{S}}$ which is the spectral separation between combined beams. $\mathrm{P}_{\mathrm{B}}$ and $\mathrm{P}_{\mathrm{T} 1}$ are the optical powers at the Bragg wavelength and transmission wavelength respectively.

In order to optimize beam combining efficiency, the thickness, $t$, and refractive index modulation, $\delta$, are varied over a range of interest. A search for the maximum combining efficiency through the resulting data will produce the optimized values for thickness and refractive index modulation.

VBGs can be manufactured in PTR glass with a wide range of thickness, $0.5 \mathrm{~mm}$ to $25 \mathrm{~mm}$ and refractive index modulation amplitude, up to $1000 \mathrm{ppm}$ [18]. If the calculation from equation (6) above were to be made over this range, the VBG design could be determined for the best possible beam combining efficiency.

Initially, the case of one VBG with one reflecting beam and one transmitting beam will be considered. Then the effects of divergent beams, beams with finite bandwidth, and losses in the glass will be considered in later sections. The same method can then be adapted to three or more beams interacting with one VBG, and, through an iterative process, to multiple VBGs in an SBC system with an arbitrary number of beams.

Figure 3 shows a contour plot of the beam combining efficiency, $\eta_{\mathrm{BC}}$ of two monochromatic plane waves interacting with a single VBG over a feasible range of thickness and refractive index modulation values for a chosen spectral separation between the beams, $0.25 \mathrm{~nm}$ in this case. The plot 
shows waves of efficient beam combining with multiple peaks and valleys as a function of thickness and refractive index modulation. These peaks in combining efficiency represent the particular thickness required for the chosen transmission wavelength to fall in one of the local minima of the VBG diffraction efficiency spectrum, shown in Figure 1 (blue dots) such that it has a lossless transmission through the VBG. The combining efficiency maximum with the thinnest grating value represents the solution for the transmitting beam to fall on the first zero of the diffraction spectrum. Thicker gratings have narrower spectral bandwidth causing the transmitting beam to lose then gain transmission efficiency until the beam falls in higher order zeros of the diffraction spectrum. This behavior results in the subsequent efficiency peaks at thicker grating values that correspond to placing the adjacent channel in the next zero of the diffraction spectrum. The diffracting beam however must be diffracted at the Bragg wavelength with maximum possible efficiency. The expression for the peak in diffraction efficiency at the Bragg wavelength for a particular VBG is given in Ref. [17]

$$
\eta_{0}=\tanh ^{2}\left(\frac{\pi t \delta n}{\lambda_{0}\left|\cos \left(\theta_{m}\right)\right|}\right)
$$

Each of the peaks in combining efficiency in Figure 3 has large areas in the thickness-refractive index modulation plane which have higher than $99 \%$ combining efficiency. If the VBGs are interacting with monochromatic plane waves, then only a small advantage is obtained by using a VBG with higher thickness or higher refractive index modulation or both due to the fact that the diffraction efficiency at the Bragg wavelength is higher. However in this case, the efficiency of the transmitting beam is unaffected if the particular thickness of the VBG is chosen such that the transmitting beam falls into one of the local minimums of the VBG diffraction efficiency profile and therefore a local maximum in beam combining efficiency.

\section{Optimization of Single Stage SBC Considering Finite Divergence}

Finite beam spectral bandwidth and divergence play an important role in the beam combining performance of a VBG, as described in Ref. [17]. To include the effects of beam divergence and spectral bandwidth on the performance of a VBG, a normalized double convolution should be performed between the diffraction efficiency of equation (5) above, and the beam's Gaussian profiles in both angle and spectral space given by equations (8) and (9):

$$
\begin{gathered}
G_{1}(\Delta \lambda, w)=e^{-2\left(\frac{\Delta \lambda-\lambda_{0}}{w}\right)^{2}} \\
G_{2}\left(\Delta \theta_{m}, \theta_{\text {Beam }}\right)=e^{-2\left(\frac{\Delta \theta_{m}-\theta_{m}}{\theta_{\text {Beam }}}\right)^{2}}
\end{gathered}
$$

where $w=$ the spectral bandwidth and $\theta_{\text {Beam }}=$ the angular divergence. The resulting diffraction efficiency spectra for beams with finite divergence and spectral bandwidth are shown in Figure 1. The plot shown is for a VBG with thickness of $4.7 \mathrm{~mm}$, refractive index modulation of $320 \mathrm{ppm}$. One can see that the effect of finite spectral width is very similar to that of finite angular divergence and it will not be shown in this paper.

The goal of an optimization is to maximize total efficiency with respect to both the transmitting and reflecting beams. Given a finite beam divergence, the diffraction efficiency at the Bragg wavelength will be reduced from the ideal case, which will reduce the power of the reflected beam. The transmission for a beam propagating through one of the non-zero minima in the diffraction efficiency spectrum will also be reduced.

An increase in either the VBG thickness or the refractive index modulation will have the effect of increasing the peak diffraction efficiency at the Bragg wavelength. The down side of this approach is that in the case of increased refractive index modulation, the transmission at the minima in the diffraction efficiency spectrum is also reduced. This is a result of the increasing overlap in angle space of the beam with finite divergence and the VBG diffraction efficiency side lobes.

The optimal thickness and refractive index modulation for a VBG intended to diffract one beam, and transmit another beam at some specific spectral separation must be found such that a balance between increasing diffraction efficiency at the Bragg wavelength and decreasing diffraction efficiency at a desired local minimum is achieved. To optimize a single stage SBC system considering beam divergence, the same approach described in the monochromatic plane-wave section is used with the addition that the diffraction efficiency spectrum, $\eta_{d \theta}\left(\lambda_{T}, t, \delta \mathrm{n}, \theta_{\text {Beam }}\right)$, used in the calculation for beam combining efficiency from equation (6) considers beam divergence by a convolution of the beam and the VBG diffraction efficiency in angle space.

To explore basic characteristics of beam combining efficiency, let us consider a situation where the spectral width of a beam is small compared to spectral selectivity of VBGs. This means that even for a very dense channel separation of $0.25 \mathrm{~nm}$, the spectral selectivity of a grating should be about $0.1 \mathrm{~nm}$ and the spectral width of a laser line should be below $0.02 \mathrm{~nm}$. This requirement is completely acceptable for narrow band fiber lasers that are typically used for spectral beam combining. If such lasers are used, then the bandwidth of the lasers has a negligible effect on the VBG performance, and can be neglected. However, even diffraction limited beam divergence of sub-10 mm diameter beams can have an effect on the VBG diffraction efficiency spectrum, and must be taken into account.

So, for the demonstration of the concept, it will be assumed that the input lasers are single frequency, and therefore the diffraction efficiency calculation and convolution in angle space can be made for only the specific wavelengths at which the lasers operate. Furthermore, it is not necessary to perform the convolution in angle space for a broad angle range. If, the calculated angle range is reduced to three times the full width of the beam divergence, the calculation matrix can be reduced significantly. s.

\section{D.Material Losses in PTR Glass}

Another important factor in optimization is to include losses associated with increasing thickness and refractive index 
modulation. Additional scattering and absorption have been observed in PTR glass after UV exposure and thermal development [19]. Additional absorption is the result of different types of defects generated in PTR glass while scattering is the result of precipitation of crystalline phase of $\mathrm{NaF}$ which has a different refractive index from that of PTR glass. It was found that scattering losses are at least one order of magnitude higher than absorption, and for the purposes of determining efficiency of SBC, $\eta_{B C}$, only scattering losses will be considered. It was shown in Ref. [20] that refractive index change $(\Delta \mathrm{n})$ is proportional to concentration of crystalline phase precipitated in glass matrix. This means that the scattering losses vary linearly with the total refractive index change $(\Delta n)$.

It was found for UV exposed and thermally developed PTR glasses that scattering losses have a $\lambda^{-4}$ spectral dependence. This result confirms that this material demonstrates Rayleigh scattering from $\mathrm{NaF}$ nano-crystals with refractive index of 1.32 compared to the 1.49 of the glass matrix. Therefore, the scattering loss coefficient (optical density divided by thickness) at any wavelength is given by the following expression.

$$
L(\lambda)_{c m^{-1}}=L\left(\lambda_{0}\right)\left(\frac{\lambda_{0}}{\lambda}\right)^{4} c m^{-1}
$$

where $\lambda_{0}$ is an arbitrary wavelength at which scattering loss, $\mathrm{L}\left(\lambda_{0}\right)$, is measured. It was found that the scattering loss at $\lambda_{0}=750 \mathrm{~nm}$ has the following empirical linear correlation with refractive index modulation ( $\delta$ ) for a typical PTR glass used for VBG recording:

$$
L\left(\lambda_{0}=750 \mathrm{~nm}\right)=200 \delta n
$$

Then the total loss in a plate with thickness $\mathrm{t}$ in $\mathrm{cm}$ can be calculated by the following equation:

$$
L_{t o t}(\lambda, t, \delta n)=\left(1-10^{-L(\lambda) t}\right)=\left(1-10^{-\frac{200 \delta n \lambda_{0}^{4} t}{\lambda^{4}}}\right)
$$

Including the scattering loss of equation(10) into the combining efficiency calculation of equation (6), yields the following expression for combining efficiency.

$$
\begin{aligned}
& \eta_{B C}(t, \delta n)=\left(\frac{1-L_{t o t}(\lambda, t, \delta n)}{P_{B}+P_{T 1}}\right) \\
& \times\left\{\begin{array}{l}
P_{B}\left[\eta_{d \theta}\left(\lambda_{B}, t, \delta n, b\right)\right] \\
+P_{T 1}\left[1-\eta_{d \theta}\left(\lambda_{T 1}, t, \delta n, b\right)\right]
\end{array}\right\}
\end{aligned}
$$

\section{E.Notable Cases for a Single Stage SBC System}

Some interesting conclusions can be made after some key optimization calculations. Up to this point, the discussion was limited to a single VBG with a single reflecting beam and a single transmitting beam with a divergence of $0.5 \mathrm{mrad}$ (half angle), and spectral separation of $0.25 \mathrm{~nm}$ between beams.
The approach can be easily expanded to an arbitrary number of transmitting beams and one reflecting beam interacting with one VBG by the following, more general equation for beam combining efficiency.

$$
\begin{aligned}
& \eta_{B C}(t, \delta n)=\left(\frac{1-L_{t o t}(\lambda, t, \delta n)}{P_{i n}}\right) \\
& \times\left\{\begin{array}{l}
P_{B}\left[\eta_{d \theta}\left(\lambda_{B}, t, \delta n, b\right)\right] \\
+P_{T_{1}}\left[1-\eta_{d \theta}\left(\lambda_{T_{1}}, t, \delta n, b\right)\right] \\
+\cdots+P_{T_{N}}\left[1-\eta_{d \theta}\left(\lambda_{T_{N}}, t, \delta n, b\right)\right]
\end{array}\right\}
\end{aligned}
$$

This equation can be written in a more concise notation:

$$
\begin{aligned}
& \eta_{B C}(t, \delta n)=\left(\frac{1-L_{t o t}}{P_{i n}}\right) \\
& \times \sum_{n=1}^{N}\left\{\begin{array}{l}
P_{B}\left[\eta_{d \theta}\left(\lambda_{B}, t, \delta n, b\right)\right] \\
+P_{T_{n}}\left[1-\eta_{d \theta}\left(\lambda_{T_{n}}, t, \delta n, b\right)\right]
\end{array}\right\}
\end{aligned}
$$

where $\mathrm{N}$ is the number of transmitting beams interacting with the VBG being optimized, $\mathrm{P}_{\mathrm{B}}$ is the power of the reflecting beam at the Bragg wavelength, $\mathrm{P}_{\mathrm{TN}}$ is the power of the Nth transmitting beam, and $\mathrm{P}_{\text {in }}$ is the total power incident on the VBG.

Equation (12) can now be used to generate a two dimensional array of VBG beam combining efficiency as a function of VBG thickness and refractive index modulation for a specified number of beams, $\mathrm{N}$, at a specified spectral separation, $\Delta \lambda_{s}$, with a specified beam divergence, $b$. An example of the resulting data for combining two beams with divergence of $0.5 \mathrm{mrad}$ and wavelengths in vicinity of $1 \mu \mathrm{m}$ with channel separation of $0.25 \mathrm{~nm}$ can be seen in Figure 4 .

The maximum combining efficiency was calculated to be $98.1 \%$ at $6.3 \mathrm{~mm}$ thickness and $170 \mathrm{ppm}$ refractive index modulation for a studied thickness range from 0 to $7 \mathrm{~mm}$ and refractive index modulation range from 10 to $600 \mathrm{ppm}$. If, however, the analysis is extended to a maximum thickness of 25 $\mathrm{mm}$, an absolute maximum combining efficiency of $98.3 \%$ can be found with a thickness of $19.9 \mathrm{~mm}$ and a refractive index modulation of $60 \mathrm{ppm}$.

The same analysis performed for a total of five beams results in a maximum of $98.5 \%$ combining efficiency at $16.8 \mathrm{~mm}$ thickness and $60 \mathrm{ppm}$ refractive index modulation. The reason that the five beam case results in a higher efficiency at a smaller thickness, is that more of the total power in the system is transmitting through the minima in the VBG spectrum rather than diffracting from the peak at the Bragg wavelength. In this case the optimization is weighted toward weaker VBGs such that the peak in the diffraction efficiency spectrum is lower, and the transmission efficiency of each of the four transmitting beams is higher. 
Referring back to Figure 4, four peaks in the combining efficiency can be seen as a function of thickness and refractive index modulation, but the peaks appear mostly in the thickness direction. As stated in the section concerning plane wave monochromatic beams, these peaks in combining efficiency represent a combination of thickness and refractive index modulation such that the transmitting beam with wavelength closest to the Bragg wavelength of the reflecting beam falls into the first, second, third, or fourth minimum of the diffraction efficiency spectrum. The solution with the thinnest grating represents the use of the first minimum, and the solution with the thickest grating represents the use of the fourth minimum. These minima can be seen in the diffraction efficiency spectrum of Figure 1.

If the optimization analysis is limited to a maximum thickness of $3 \mathrm{~mm}$, the first peak in combining efficiency can be found, and results in a maximum of $97.40 \%$ combining efficiency at a thickness of $2.1 \mathrm{~mm}$ and refractive index modulation of $470 \mathrm{ppm}$. As discussed in the previous paragraph, the first peak in combining efficiency corresponds to using a VBG thickness and refractive index modulation such that the transmitting beam with wavelength nearest to the Bragg wavelength falls in the first minimum of the diffraction efficiency spectral profile. Analysis of the second peak shows a combining efficiency of 97.97 $\%$ at a thickness of $3.4 \mathrm{~mm}$ and $\delta \mathrm{n}$ of $310 \mathrm{ppm}$, a gain of $0.57 \%$. Examining the third peak shows a combining efficiency of 98.06 $\%$ at a thickness of $4.8 \mathrm{~mm}$ and $\delta \mathrm{n}$ of $220 \mathrm{ppm}$, a gain of only 0.09 $\%$. The fourth peak shows a combining efficiency of $98.12 \%$ at a thickness of $6.3 \mathrm{~mm}$ and $\Delta \mathrm{n}$ of $170 \mathrm{ppm}$, a gain of only $0.06 \%$. These examples are specific to beam divergences of $0.5 \mathrm{mrad}$ (half angle), and single frequency lasers with $0.25 \mathrm{~nm}$ spectral separation. For beams with higher divergence, the differences in combining efficiency for successive peaks will be exaggerated, and the use of thicker gratings utilizing higher order minimums for transmission is benificial. However, in the case of collimated beams, and narrow bandwidth lasers, the gain in combining efficiency after the third peak is only $0.06 \%$. It is worth pointing out that the next contour down from the highest efficiency contour in Figure 4 is set to be only $0.5 \%$ below the peak in beam combining efficiency. So rather than there being a single solution, VBGs manufactured in the range shown by this contour level will produce reasonably high beam combining efficiency.

\section{F.Multi-Stage SBC Optimization by a Sequence of VBGs}

The optimization analysis up to this point has been with regard to a single VBG SBC system. Even for cases in which more than two beams were considered, one beam would be reflecting from one VBG, while the remaining beams were transmitting through the same VBG. A multi-beam, VBG based SBC system will have as many VBGs as beams minus one. Optimization is performed iteratively for each successive VBG in the system.

The first VBG in the SBC system will only interact with two beams, the diffracted beam at the VBG resonant wavelength, and the transmitted beam at some specified spectral separation from the resonant wavelength, illustrated in Figure 2. The next VBG in the system will interact with three beams, the diffracted beam at the VBG resonant wavelength, and the two beams from the first VBG which will both transmit through the second VBG.
This continues until the desired number of beams is combined. A four stage SBC of five beams is illustrated in Figure 5.

The calculated output of each iteration, which is the ratio of the power incident on the optimized VBG to the power that will arrive at the next VBG for each beam, is used as the input for the next iteration. The final result of the optimization is the necessary refractive index modulation and thickness for each $\mathrm{VBG}$ in the system to obtain peak combining efficiency of the entire system.

Figure 6 shows an example of the resulting data from an optimization calculation for a 5-beam combining system with a spectral separation between channels of $0.25 \mathrm{~nm}$ from $1064 \mathrm{~nm}$ to $1065 \mathrm{~nm}$, and beam divergence of $1 \mathrm{mrad}$ (half angle). The thickness and refractive index modulation parameters for the four optimized VBGs associated with the third maximum are (1) $4.89 \mathrm{~mm}$, and $240 \mathrm{ppm}$, (2) $4.73 \mathrm{~mm}, 210 \mathrm{ppm}$, (3) $4.68 \mathrm{~mm}, 200$ ppm, and (4) $4.66 \mathrm{~mm}, 200 \mathrm{ppm}$. The maximum achievable efficiency for five beam SBC is $95.3 \%$. The primary losses in this case are diffractive, but a detectable portion is caused by scattering. This model does not account for beam quality degradation through the system. In this case the BCF is equal to the system efficiency, $\mathrm{BCF}=0.953$.

The ideal diffraction efficiency for each of the four VBGs whose parameters were determined by the optimization procedure is shown in Figure 7. The diffraction efficiency spectral profile of each VBG of Figure 7 is only extended far enough to overlap with the associated interacting beams. The wavelengths of the beams are represented by vertical lines. The diffraction efficiency for the matched VBG can be observed at the top of each line, while the transmission efficiency for each of the following VBGs can be observed at the bottom of each line. Beam one does not diffract, but transmits through four VBGs, while beam five diffracts from the final VBG, and transmits through none. This system was designed such that each laser would interact with every VBG whose Bragg wavelength is less than or equal to that of the laser. All VBGs in Figure 7 have less than 1\% diffraction loss at wavelengths corresponding to the Bragg condition of higherwavelength VBGs.

\section{Experimental Demonstration of Ultimate Efficiency for High Spectral Density SBC}

The optimization technique discribed above was used to design a VBG for the following experiment with $4.7 \mathrm{~mm}$ thickness, and 200 ppm refractive index modulation, corresponding to the use of the third local maximum from Figure 4. Figure 2 shows the experimental architecture for single stage, 2 channel high power spectral beam combining. One VBG is used to combine two high power lasers.

The individual fiber lasers provide output power of $155 \mathrm{~W}$ each with spectral separation of $0.25 \mathrm{~nm}$. The beam with the lower wavelength was diffracted from the VBG while the beam with the higher wavelength was transmitted through the third minimum in the VBG's diffraction efficiency spectrum. The combined output power was $301 \mathrm{~W}$ showing 97\% beam combining efficiency, close to the theoretical limit.

As discussed in Section 2, the total increase of brightness provided by SBC is described by the BCF that includes not only 
power losses but also deterioration of beam quality. To provide the characterization, the $\mathrm{M}^{2}$ beam quality factor of the combined beam for a two channel SBC system at $300 \mathrm{~W}$ was measured to be $\mathrm{M}^{2} \mathrm{x}=1.19$, and $\mathrm{M}^{2} \mathrm{y}=1.16$, while the $\mathrm{M}^{2}$ of the individual beams was measured to be less than 1.05. Figure 8 shows the combined beam caustic for the vertical case. The radiance of the combined beam is calculated to be, $192 \mathrm{TW} /\left(\mathrm{sr} \cdot \mathrm{m}^{2}\right)$. The original radiance of each beam was $124 \mathrm{TW} /\left(\mathrm{sr}^{\prime} \mathrm{m}^{2}\right)$. This gives a $\mathrm{BCF}$ of 0.77. It is important to note that even though the combined beam has close to diffraction limit divergence, the brightness in the combined beam is lower than the double brightness of the original beams. The study of phenomena deteriorating brightness in a combined beam will be continued in the following paper.

The total spectral bandwidth of the combined beam is $0.25 \mathrm{~nm}$, therefore the spectral radiance is calculated to be $770 \mathrm{TW} /\left(\mathrm{sr}^{2} \mathrm{~m}^{2} \cdot \mathrm{nm}\right)$. To our knowledge, this is the highest spectral radiance for any spectral beam combining system demonstrated to date.

\section{Conclusions}

Spectral beam combining by volume Bragg gratings recorded in photo-thermo-refractive glass is a powerful tool for laser applications which require higher radiance than a single laser unit can achieve. The beam combining factor (BCF) that describes the change of radiance provided by a beam combining system and is not affected by the individual beam quality of the input lasers has been introduced as a tool to compare various beam combining methods and experiments. A method of optimization of VBGs which provides a maximum efficiency of spectral beam combining has been described for an arbitrary number of beams. Previous demonstrations of multistage SBC have utilized a set of VBGs which were optimized considering only a single stage $[7,8]$. It is shown in this paper that improved efficiency can be achieved by optimizing each VBG in a multistage SBC system independently. An experiment confirming the proposed modeling for single stage SBC has demonstrated a total combined power of $301 \mathrm{~W}$ with channel separation of $0.25 \mathrm{~nm}$, efficiency of combining $97 \%$, close to diffraction limit divergence with $\mathrm{M}^{2} \mathrm{x}=1.19$, and $\mathrm{M}^{2} \mathrm{y}=1.16$, beam combining factor $(\mathrm{BCF})$ of 0.77 , and spectral radiance of $770 \mathrm{TW} /\left(\mathrm{sr} \cdot \mathrm{m}^{2} \cdot \mathrm{nm}\right)$, the highest to date for spectrally combined beams.

\section{Acknowledgements}

This research was funded by the Defense Advanced Research Projects Agency (DARPA), ADHELS Program; contract number H0011-06-1-0010. D. Drachenberg acknowledges the support of the Directed Energy Professional Society (DEPS) Graduate Scholarship.

\section{References}

1. T. Y. Fan, "Laser beam combining for high-power, high-radiance sources," IEEE Journal of Selected Topics in Quantum Electronics 11, 567-577 (2005).
2. C. Wirth, O. Schmidt, I. Tsybin, T. Schreiber, R. Eberhardt, J. Limpert, A. Tünnermann, K. Ludewigt, M. Gowin, E. ten Have, and M. Jung, "High average power spectral beam combining of four fiber amplifiers to $8.2 \mathrm{~kW}$.," Optics Letters 36, 3118-20 (2011).

3. S. J. Augst, J. K. Ranka, T. Y. Fan, and A. Sanchez, "Beam combining of ytterbium fiber amplifiers (Invited)," Journal of the Optical Society of America B 24, 1707 (2007).

4. S. Sugimoto and K. Minemura, "High-speed digitalsignal transmission experiments by optical wavelength-division multiplexing," Electronics ... 13, 680-682 (1977).

5. O. Schmidt, C. Wirth, D. Nodop, J. Limpert, T. Schreiber, T. Peschel, R. Eberhardt, and A. Tünnermann, "Spectral beam combination of fiber amplified ns-pulses by means of interference filters.," Optics Express 17, 22974-82 (2009).

6. X. Chu, S. Zhao, L. Shi, S. Zhan, J. Xu, and Z. Wu, "Expansion of the channel number in spectral beam combining of fiber lasers array based on cascaded gratings," Optics Communications 281, 4099-4102 (2008).

7. A. Sevian, O. Andrusyak, I. Ciapurin, V. Smirnov, G. Venus, and L. Glebov, "Efficient power scaling of laser radiation by spectral beam combining.," Optics Letters 33, 384-386 (2008).

8. O. Andrusyak, V. Smirnov, G. Venus, and L. Glebov, "Beam combining of lasers with high spectral density using volume Bragg gratings," Optics Communications 282, 2560-2563 (2009).

9. Z. Sheng-bao, Z. Shang-hong, C. Xing-chun, W. Zhuo-liang, and S. Lei, "Spectral beam combining of fiber lasers based on a transmitting volume Bragg grating," Optics \& Laser Technology 42, 308-312 (2010).

10. T. Y. Fan and A. Sanchez, "Coherent (phased array) and wavelength (spectral) beam combining compared (Invited Paper)," in Fiber Lasers II: Technology, Systems, and Applications, L. N. Durvasula, A. J. W. Brown, and J. Nilsson, eds. (SPIE, 2005), Vol. 5709, pp. 157-164.

11. L. Glebov, "Volume diffractive elements in photosensitive inorganic glass for beam combining," in Proceedings of Solid State and Diode Lasers Technical Review (2001), p. FA-5. 
12. H. Kogelnik, "Coupled wave theory for thick hologram gratings," The Bell System Technical Journal 48, 2909-2947 (1969).

13. L. B. Glebov, J. Lumeau, S. Mokhov, V. Smirnov, and B. Y. Zeldovich, "Reflection of light by composite volume holograms: Fresnel corrections and Fabry-Perot spectral filtering," Journal of the Optical Society of America A 25, 751-764 (2008).

14. I. V. Ciapurin, L. B. Glebov, and V. I. Smirnov, "Modeling of phase volume diffractive gratings, part 1: transmitting sinusoidal uniform gratings," Optical Engineering 45, 015802-9 (2006).

15. O. M. Efimov, L. B. Glebov, L. N. Glebova, K. C. Richardson, and V. I. Smirnov, "High-Efficiency Bragg Gratings in Photothermorefractive Glass," Applied Optics 38, 619 (1999).

16. M. McCall, "Axial electromagnetic wave propagation in inhomogeneous dielectrics *1," Mathematical and Computer Modelling 34, 14831497 (2001).

17. I. V. Ciapurin, D. R. Drachenberg, V. I. Smirnov, G. B. Venus, and L. B. Glebov, "Modeling of phase volume diffractive gratings, part 2: reflecting sinusoidal uniform gratings, Bragg mirrors," Optical Engineering 51, 058001 (2012).

18. L. B. Glebov, "Volume Holographic Elements in a Photo-Thermo-Refractive Glass," Journal of Holography and Speckle 5, 77-84 (2009).

19. L. B. Glebov, "Photochromic and Photo-ThermoRefractive Glasses," in Encyclopedia of Smart Materials. (2002).

20. T. Cardinal, O. M. Efimov, H. G. Francois-SaintCyr, L. B. Glebov, L. N. Glebova, and V. I. Smirnov, "Comparative study of photo-induced variations of $\mathrm{X}$-ray diffraction and refractive index in photothermo-refractive glass," Journal of NonCrystalline Solids 325, 275-281 (2003).

21. J. W. Strutt, "On the Blue Light from the Sky, Its Polarization and Colour," Philisophical Magazine Series 4 41, 107-119 (1871). 
Figures and Captions

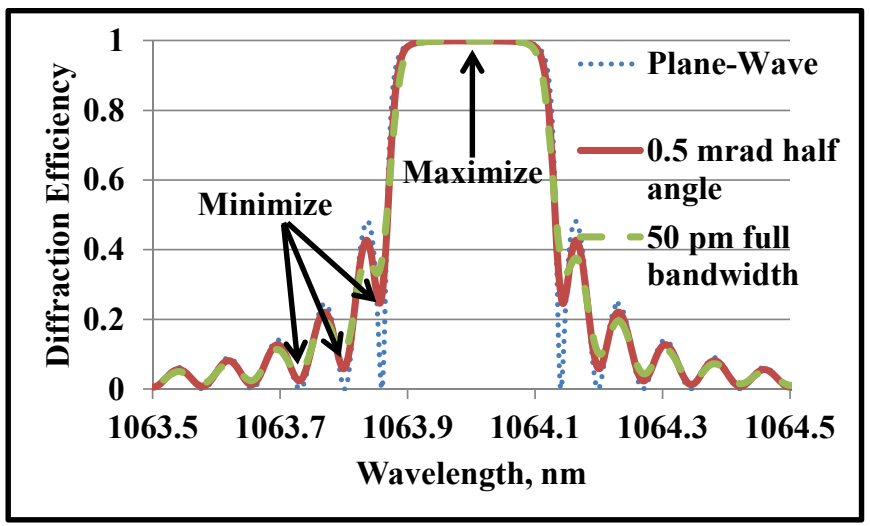

Figure 1: Diffraction efficiency spectrum of a reflecting VBG with following parameters: $\mathrm{t}=4.7 \mathrm{~mm}$, and $\delta \mathrm{n}=320 \mathrm{ppm}, \mathrm{n}_{\text {aver }}=1.486, \mathrm{~d}=358.9 \mathrm{~nm}$, Bragg angle in air $4^{\circ}$ for $1064 \mathrm{~nm}$. Probe beam: monochromatic plane wave (bluedots), monochromatic divergent wave $\left(\theta=0.5 \mathrm{mrad}\right.$, half angle at $\left.1 / \mathrm{e}^{2}\right)(\mathrm{red}$ solid), polychromatic plane wave with $50 \mathrm{pm}$ full spectral width (green dash).

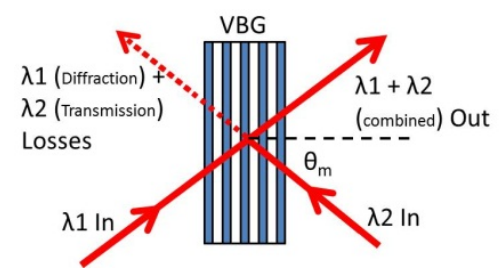

Figure 2: Illustration of a single stage SBC of two beams using a single reflecting VBG. Beam $1(\lambda 1)$ transmits through a VBG diffraction minimum with some diffraction loss. Beam 2 ( $(12)$ diffracts at the Bragg condition with some transmission loss.

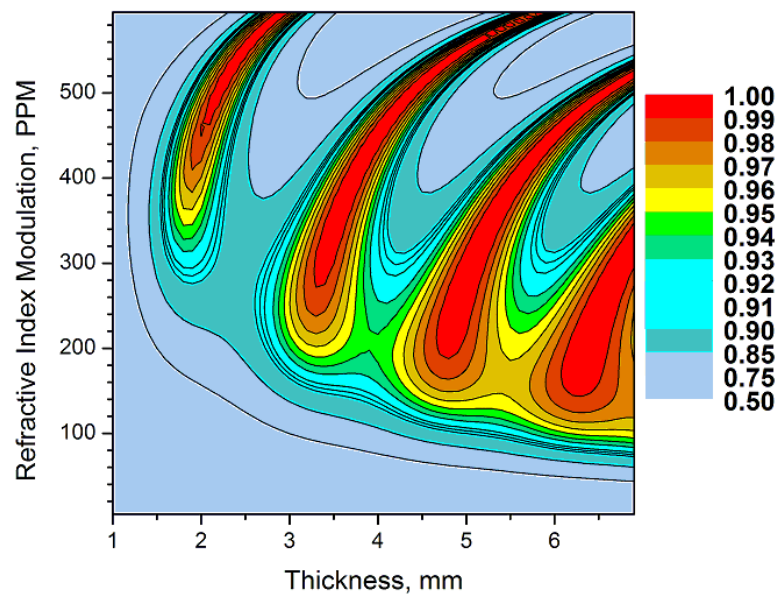

Figure 3: Combining efficiency of monochromatic plane waves combined by a single VBG (material losses are not considered) versus thickness and refractive index modulation.

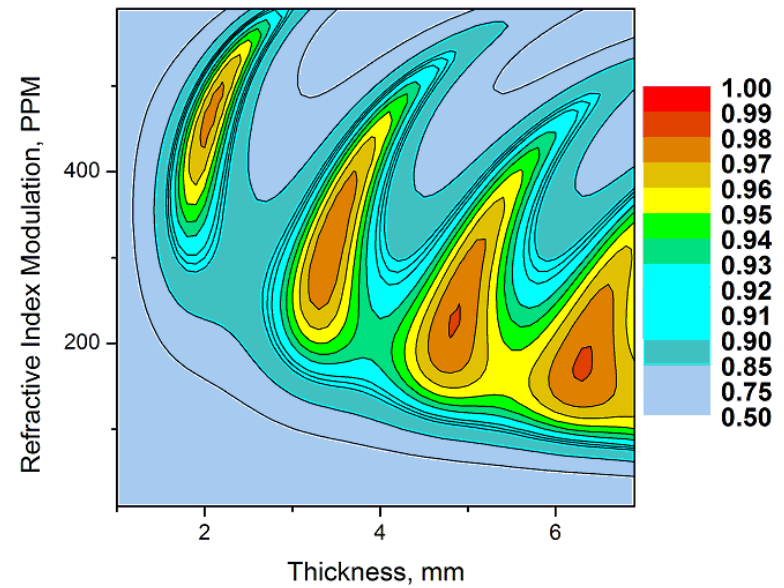

Figure 4: Combining efficiency of 2 beams by a single VBG with $0.25 \mathrm{~nm}$ spectral separation and $0.5 \mathrm{mrad}$ divergence including the effects of material losses.

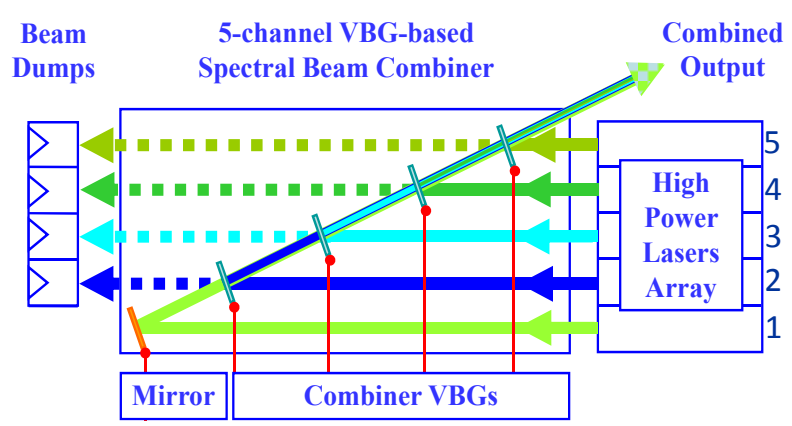

Figure 5: Illustration of four stage SBC of five beams. Numbers correspond to those in Figure 7

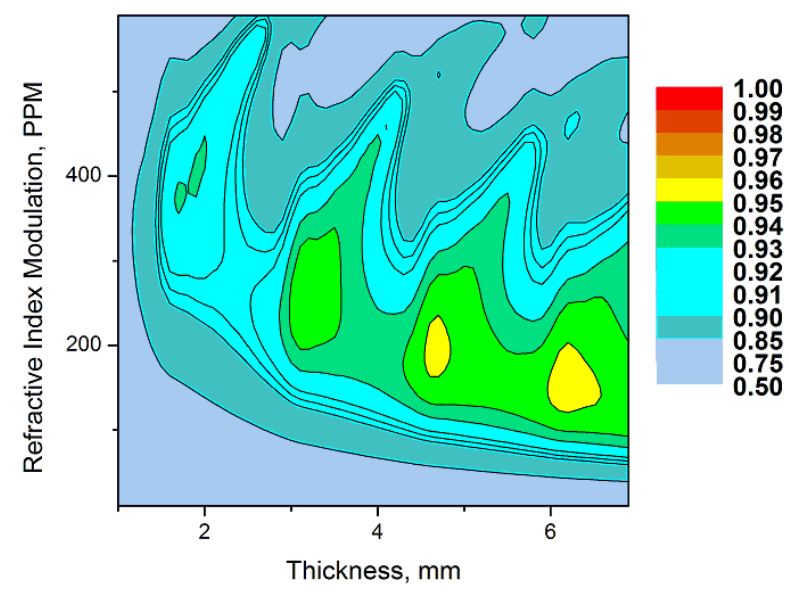

Figure 6: Efficiency of 5-channel SBC by four VBGs as a function of VBG thickness and refractive index modulation for $0.25 \mathrm{~nm}$ spectral separation, and $0.5 \mathrm{mrad}$ of divergence including the effects of material losses. 


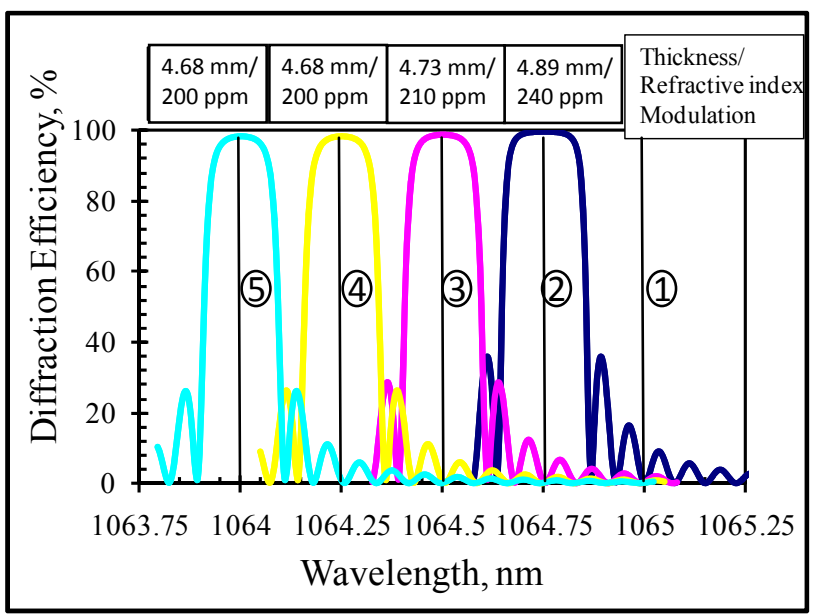

Figure 7: Diffraction efficiency spectra of VBGs for an optimized 5-channel system. Vertical solid lines represent the wavelengths of five combined beams, where beam 1 transmits through all four VBGs, and beam 5 diffracts off of the final VBG. The diffraction efficiency spectrum of each VBG is only extended far enough to overlap with the associated interacting beams. Numbers correspond to beams in Figure 5 .

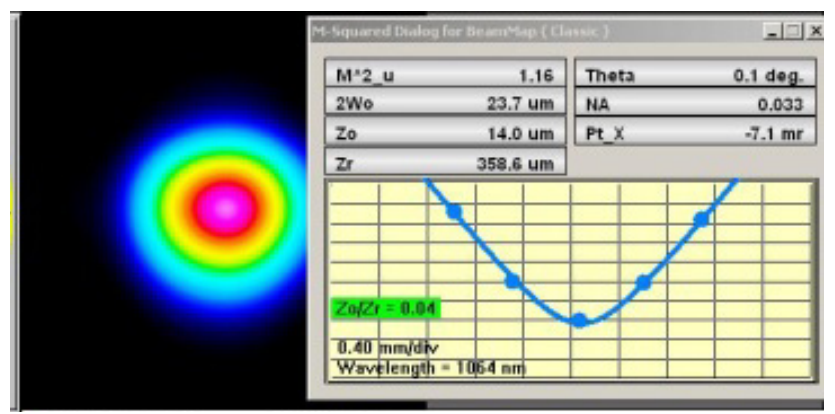

Figure 8: 2-Beam, $300 \mathrm{~W}, \mathrm{SBC} \mathrm{M}^{2}$ 